\title{
Computational Design of TrkB Peptide Inhibitors and Their Biological Effects on Ovarian Cancer Cell Lines
}

\author{
Marzieh kafshdouziamin ${ }^{1}$ Mansour Poorebrahim ${ }^{2}$ - Saeideh Najafi ${ }^{3}$. \\ Hamzeh Rahimi ${ }^{4}$. Morteza Karimipoor ${ }^{4} \cdot$ Nematollah Gheibi $^{5}$
}

Accepted: 29 December 2015/Published online: 7 January 2016

(C) Springer Science+Business Media New York 2016

\begin{abstract}
There are large numbers of different intracellular signaling pathways regulated by Tyrosine kinases (Trk) receptors. Trk receptors, especially TrkB, are also frequently overexpressed in a variety of human malignant tumors. In this study, we have computationally designed small peptide-based inhibitors of TrkB and investigated their effects on the proliferation and apoptosis of two ovarian cancer cell lines. Molecular docking of TrkB with its ligand and antagonist, BDNF and Cyclotraxin B respectively, was carried out using HADDOCK program. A peptide library was constructed based on the critical residues involved in the TrkB binding site. After docking and optimization, two selected peptides were purchased and their effects on the viability and apoptosis of the cells were evaluated by performing MTT (3-[4,5-dimethylthiazol-2-yl]-2,5-diphenyltetrazolium bromide) test and flow cytometry assay. Subsequently, the levels of expression and phosphorylation statues of TrkB and its two downstream genes including MAPK3 and eIF4E were assessed
\end{abstract}

Nematollah Gheibi

ngheibi@qums.ac.ir

1 Department of Medical Biotechnology, Para Medicine School, Qazvin University of Medical Sciences, Qazvin, Iran

2 Department of Medical Biotechnology, School of Advanced Technologies in Medicine, Tehran University of Medical Sciences, Tehran, Iran

3 Department of Microbiology, Faculty of Biology Sciences, Islamic Azad University of Tonekabon Branch, Tonekabon, Iran

4 Molecular Medicine Department, Biotechnology Research Center, Pasteur Institute of Iran, Tehran, Iran

5 Cellular and Molecular Research Center, Qazvin University of Medical Sciences, Qazvin, Iran with western blot. We found that designed peptides effectively reduced TrkB, MAPK3 and eIF4E phosphorylation, reduced cell viability and induced apoptosis in the treated cells when compared to untreated cells. In conclusion, the BDNF/TrkB signaling is shown to be attenuated substantially in the presence of peptide inhibitors suggesting a strong inhibitory potential of the designed peptides for Trk family.

Keywords TrkB $\cdot$ Cancer $\cdot$ Small peptide $\cdot$ Docking

\section{Introduction}

The Tyrosine kinases (Trk) receptor family is one of the best-known class of transmembrane receptors that is necessary for development and survival of the mammalian nervous system. There are three most common types of Trk receptors including TrkA, TrkB, and TrkC which have different binding affinity to certain types of neurotrophins (Nakagawara 2001). Comparisons of their function indicate that TrkB and TrkC are the receptors for BDNF, NT4 and NT3 respectively, while TrkA is activated by nerve growth factor (NGF). These tyrosine kinase receptors are highly related to different intracellular signaling pathways regulating proliferation, cell survival, axonal and dendritic growth, synapse formation, cytoskeleton remodeling and membrane trafficking (Huang and Reichardt 2003). It has been widely demonstrated that Trk receptors activate both MAPKs and Akt, leading ultimately to further downstream signaling pathways for cell proliferation and development (Jang et al. 2007). However, many studies have reported that skewed population of neurons expresses TrkB, which suggests that this receptor plays a critical role in the regulation of neuronal development in vivo ( $\mathrm{Li}$ et al. 2012). 
Furthermore, other important studies underline the role of TrkB in the invasion ability of cancer cells. Ectopic expression of TrkB and its ligand, BDNF, has been shown to be associated with aggressive tumor behavior and poor prognosis in cancer patients (Okamura et al. 2012). TrkB is also overexpressed by some tumors of the ovarian cancer (Yu et al. 2008), lung cancer (Götz and Sendtner 2014), colorectal cancer (Dawson et al. 2014), neuroblastoma, prostate adenocarcinoma, Wilm's tumors, pancreatic adenocarcinoma and myeloma, and is linked to an increased in anti-cancer drug resistance and tumor invasion (Thiele et al. 2009). Previously, Fujikawa et al. (2012) demonstrated that high TrkB expression is accompanied by epithelial-to-mesenchymal transition (EMT) in colorectal cancer resulting in enhanced tumor metastasis (Fujikawa et al. 2012). Accordingly, TrkB receptor can be considered as a promising target in attempts to inhibit tumor invasion and metastasis. Based on the Trk receptors, some strategies have been proposed for drug discovery to overcome chemotherapy resistance in Trk-associated cancers, but these efforts have not yet been very successful (Maina 2014). Recently, Cazorla et al. designed a potent anti-TrkB inhibitor by using peptidomimetic approach. This small peptide, Cyclotraxin B, could sufficiently alter the conformation of TrkB receptor resulting in the inhibition of BDNF/TrkB signaling and its downstream targets (Cazorla et al. 2010). This study demonstrates that TrkB targeting using peptide-based inhibitors can considerably modulate BDNF/TrkB pathway resulting in a decreased function of proliferation-related genes in TrkB overexpressed cancer cells.

Small peptide-based inhibitors are very interesting candidates for cancer therapy because of their unique physicochemical properties (Wu et al. 2014). TrkB is a well-characterized protein which synthesized as single-pass transmembrane and has been recently considered as a potentially suitable receptor for designing new small peptide antagonists (Cazorla et al. 2010). Although inhibition of TrkB receptor may effectively reduce cancer invasion during cancer progression, most studies have been focused on the TrkB activation in the neural disorders through small-molecule agonists (Jiang et al. 2013; Zeng et al. 2013).

In this study, we designed several BDNF-derived small peptides using molecular docking studies and evaluated their effects on the proliferation and apoptosis of TrkB overexpressed ovarian cancer cells. These peptides were designed based on the binding site of BDNF located at the variable region III of BDNF. Among five top peptides, two peptides were chosen for experimental analysis. These peptides, PEP1 and PEP2, could bind to and significantly inhibit TrkB activity as well as its two downstream genes, MAPK3 and eIF4E, in the ovarian cancer cell lines.

\section{Materials and Methods}

\section{In Silico Design of Peptide Inhibitor Using Molecular Docking}

A library of the TrkB small peptides was built corresponding to the region III of the BDNF with sequence of

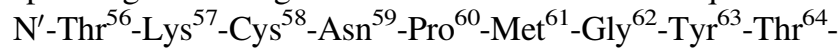
Lys $^{65}$-Glu ${ }^{66}-C^{\prime}$ using HADDOCK software (De Vries et al. 2007). Preparing of the 3D structures was the starting point for the modeling studies. The crystal structure of both TrkB (PDB code: $1 \mathrm{WWB}$ ) and BDNF (PDB code: 1B8M) proteins obtained from PDB database (www.rcsb.org). Prior to docking, the 3D structures of proteins were visualized by PyMOL program and unwanted molecules were removed from the PDB files (http://pymol.sourceforge.net). After the study of the interaction of TrkB with BDNF and Cyclotraxin B, the key residues involved in the interaction were determined and provided as input to the Rosetta software for designing new peptides (Rohl et al. 2004). We used the Backrub for flexible peptide-backbone modeling and sequence tolerance protocol for designing the stable peptides. Finally, the models with high scores were selected using the R software (2012). During the backrub protocol many mutations were generated that need to be sorted based on energy score. In order to identify the most stable sequences, we used R software for sorting generated ensembles. The 3D structure of selected peptides was modeled using PEP-FOLD modeling program (Maupetit et al. 2009). Molecular docking studies were carried out using HADDOCK software and schematic representation of docked complexes were generated using the LIGPLOT program (Wallace et al. 1995).The energy value of docked peptides was evaluated and eventually, two peptides with lower energy than others were selected and synthesized by TAG Copenhagen (Copenhagen, Denmark).

\section{Molecular Dynamics Simulation}

Molecular dynamics (MD) simulation was carried out using GROMACS (version 4.5) software package (Pronk et al. 2013). The 3D structures of peptide-TrkB complexes were solvated in a solvation box with $10 \AA$ distance between the edges of the box and the protein fragments. In order to neutralize the system, $\mathrm{Na}^{+}$and $\mathrm{Cl}^{-}$ions were added into the system. The equilibrated systems were subjected to $10 \mathrm{~ns}$ MD simulation in the isothermal-isobaric (NPT) ensemble using the leap-frog algorithm with an integration time step of 0.002 ps.

\section{Cells and Cell Culture}

The SK-OV-3 and OV-CAR-3 cell lines were purchased from American Type Culture Collection (Manassas, VA). 
The cells were maintained in RPMI 1640 supplemented with $10 \%$ FBS and $10 \mu \mathrm{g} / \mathrm{ml}$ penicillin. Both cell lines were seeded in their respective growth media and cultured in a humidified atmosphere of $5 \% \mathrm{CO}_{2}$ and $95 \%$ air at $37{ }^{\circ} \mathrm{C}$.

\section{Cell Growth Assay}

The cytotoxic effect of inhibitory peptides on the SK-OV-3 and OV-CAR-3 cancer cell lines was measured using MTT dye reduction assay. The cells $\left(7 \times 10^{4}\right.$ cells $/ 100 \mu \mathrm{l}$ media) were seeded in 96-well plates and were grown for $24 \mathrm{~h}$. Then, cells were incubated with different concentrations of the inhibitory peptides $(50,200,350$ and $500 \mathrm{nM})$ for 24,48 and $72 \mathrm{~h}$. The plates were then incubated with $100 \mu \mathrm{l}$ of $0.5 \mathrm{mg} / \mathrm{ml}$ MTT solution for $4 \mathrm{~h}$ at $37{ }^{\circ} \mathrm{C}$. The formed formazan crystals were dissolved by the addition of $100 \mu \mathrm{l} /$ well DMSO and the absorbance was measured at $570 \mathrm{~nm}$ by a 96 -well micro plate ELISA reader. Data were presented as relative percent viability with respect to untreated and Cyclotraxin B-treated cells. The $\mathrm{IC}_{50}$ was calculated from mean $\pm \mathrm{SD}$ values.

\section{Apoptosis Analysis}

After an overnight incubation, cells were treated with $200 \mathrm{nM}$ control vehicle (Cyclotraxin B) and various concentrations ( 350 and $450 \mathrm{nM}$ ) of inhibitory designed peptides for 24 and $48 \mathrm{~h}$. After treatment, cells were stained with FITC-Annexin V using FITC-Annexin V Detection kit (Biolegend) followed by flow cytometry. Subsequently, samples were washed twice with PBS, resuspended in Annexin-V binding buffer, stained with PI $(50 \mathrm{mg} / \mathrm{ml})$ and kept in the dark for $15 \mathrm{~min}$ at $20-25^{\circ} \mathrm{C}$. Staining was measured on a FACScan and percentages of apoptotic cells were determined using FACSDiva software (BD Biosciences).

\section{Analysis of TrkB Downstream Signaling Pathways}

It has been reported that $\operatorname{TrkB}$ is overexpressed more highly in OV-CAR-3 than SK-OV-3 cells (Yu et al. 2008), therefore we decided to evaluate peptides effect on the TrkB signaling pathway in OV-CAR-3 cell line. OV-CAR3 cells were grown, treated with $350 \mathrm{nM}$ PEP1, PEP2 and Cyclotraxin B for $48 \mathrm{~h}$ and lysed in Passive Lysis Buffer. Lysates were centrifuged at $13000 \times g$ for $10 \mathrm{~min}$ at $4{ }^{\circ} \mathrm{C}$. Theproteins in the lysate were fractionated on $10 \%$ SDS-PAGE gel, transferred onto PVDF membrane, washed, and blocked in TBS with $3 \%$ BSA. Immunodetection was carried out using specific primary antibodies: TrkB, phospho-TrkB, MAPK3, phospho-MAPK3, eIF4E, phospho-eIF4E (1/1000), and $\beta$-actin $(1 / 20,000)$. The HRP- conjugated secondary antibodies were used at 1:2500 dilutions. All specific primary and secondary antibodies were purchased from GenScript (Piscataway, NJ) and Cell signaling Inc respectively. Untreated cells were taken as negative control and $\beta$-actin was used for loading control. The blots were processed with the ECL Plus Western Blotting detection kit (Pierce Biotechnology, Rockford, IL, USA) and the signal detected using an LAS-3000 image analyzer (Fuji Photo Film Co., Tokyo, Japan). Quantification of Western blots was performed using ImageJ software (Rasband 2012). The statistical analyses were performed with SPSS 15.0 software (SPSSInc, Chicago, Illinois) using Student's $t$ tests toanalyze statistical significance.

\section{Statistical Analysis}

All experiments were done in triplicate, statistical analysis was performed by using one-way ANOVA and Tukey's post hoc tests $(\alpha=0.05)$.

\section{Result}

\section{Peptide Library Construction}

A peptide library of random amino acid sequences was constructed based on genetic algorithm. After generating the peptide library using backrub and sequence tolerance protocols, the five most stable peptides were selected based on the energy scores in the $\mathrm{R}$ package and subjected to HADDOCK docking program (data not shown). The molecular weight, isoelectric point, instability and other properties of selected peptides were predicted using MolProbility and ProtParam web tools (Table 1).

\section{Study of Molecular Docking of Designed Peptides with TrkB}

Initially, 3D structure of selected peptides was predicted using PEP-FOLD modeling software followed by energy minimization (Fig. 1). Automated docking of peptides with TrkB performed using the standard HADDOCK protocol and the energy value of each peptide calculated and listed in Table 2. After docking with TrkB, PEP2 had the lowest energy value among all six analyzed peptides.

The residues of five selected peptides involved in polar and non-polar interactions with TrkB were illustrated using LIGPLOT and tabulated in Table 3. Comparative analysis of HADDOCK result indicated that the designed PEP1 (TGLDSDGLYQN) and PEP2 (NTDLLNSNDNG) had the highest affinity for TrkB among five selected peptides. Therefore, these two peptides were selected for experimental analyses. Molecular docking indicated that PEP1, 
Table 1 Predicted properties of five selected peptides using Molprobility and Protparam servers

\begin{tabular}{|c|c|c|c|c|c|c|c|}
\hline Peptide name & Peptide sequence & MW & TPI & No. NAA & No. PAA & Chemical formula & GRAVY \\
\hline PEP1 & TGLDSDGLYQN & 1182.2 & 3.56 & 2 & 0 & $\mathrm{C}_{49} \mathrm{H}_{75} \mathrm{~N}_{13} \mathrm{O}_{21}$ & -0.909 \\
\hline PEP2 & NTDLLNSNDNG & 1176.1 & 3.56 & 2 & 0 & $\mathrm{C}_{45} \mathrm{H}_{73} \mathrm{~N}_{15} \mathrm{O}_{22}$ & -1.391 \\
\hline PEP3 & QYQNNQATLDQ & 1322.3 & 3.80 & 1 & 0 & $\mathrm{C}_{54} \mathrm{H}_{83} \mathrm{~N}_{17} \mathrm{O}_{22}$ & -1.900 \\
\hline PEP4 & SSTQDTRQNTS & 1224.2 & 5.55 & 1 & 1 & $\mathrm{C}_{45} \mathrm{H}_{77} \mathrm{~N}_{17} \mathrm{O}_{23}$ & -2.091 \\
\hline PEP5 & DEKFKKQYFYL & 1508.7 & 8.38 & 2 & 3 & $\mathrm{C}_{74} \mathrm{H}_{105} \mathrm{~N}_{15} \mathrm{O}_{19}$ & -1.400 \\
\hline Cyclotraxin B & CNPMGYTKEGC & 1271.4 & 7.85 & 1 & 2 & $\mathrm{C}_{53} \mathrm{H}_{86} \mathrm{~N}_{14} \mathrm{O}_{18} \mathrm{~S}_{2}$ & -1.373 \\
\hline
\end{tabular}

$M W$ molecular weight, TPI theoretical pI, No. NAA number of negative amino acids, No. PAA number of positive amino acids, GRAVY grand average of hydropathicity
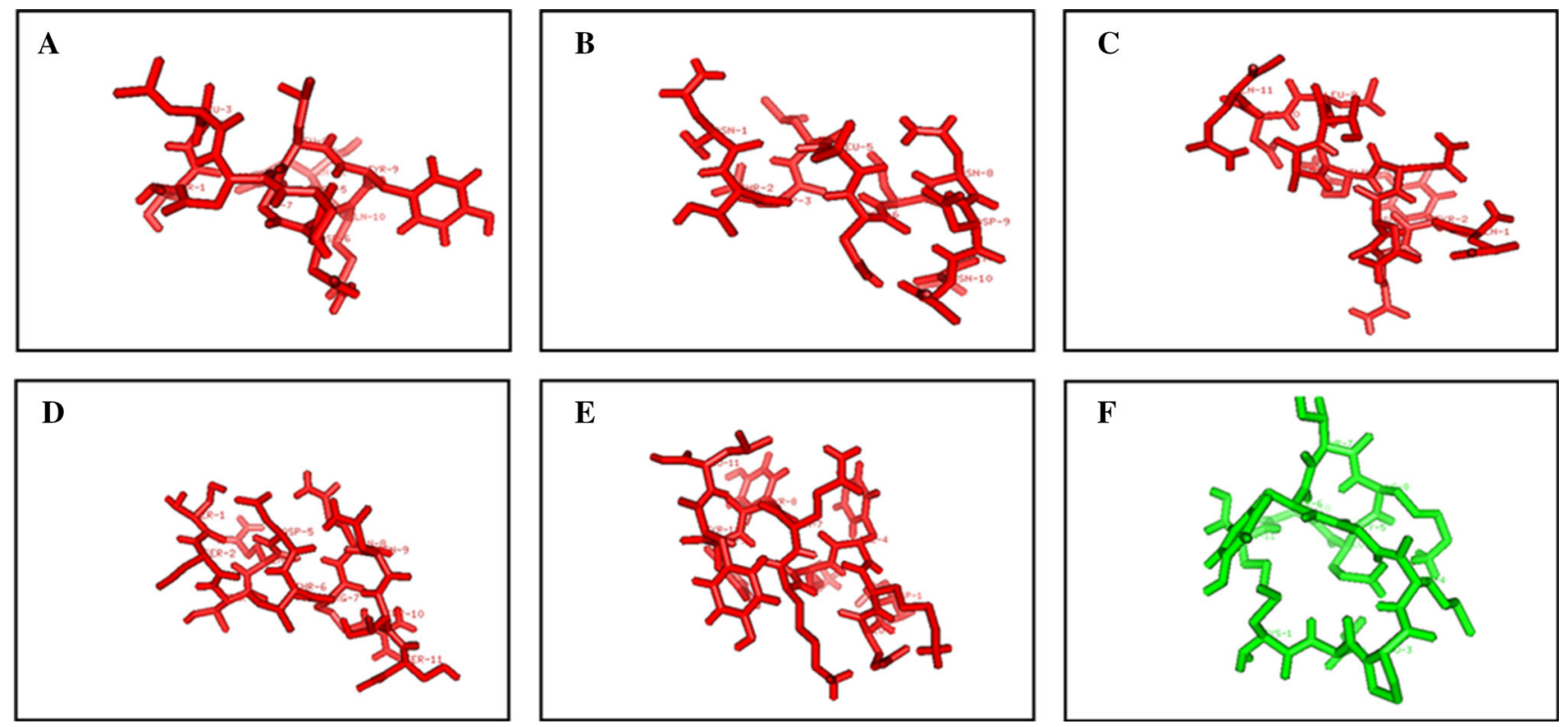

Fig. 1 Predicted structures of five selected peptides and Cyclotraxin B using PEP-FOLD tool. a PEP1, b PEP2, c PEP3, d PEP4, e PEP5, f Cyclotraxin B

Table 2 Docking energy values of selected peptides with TrkB using HADDOCK

\begin{tabular}{llll}
\hline Protein & Peptide name & Peptide sequence & Energy $(\mathrm{kcal} / \mathrm{mol})$ \\
\hline TrkB & PEP1 & TGLDSDGLYQN & -36.8 \\
& PEP2 & NTDLLNSNDNG & -47.9 \\
& PEP3 & QYQNNQATLDQ & -20.4 \\
& PEP4 & SSTQDTRQNTS & -12.5 \\
& PEP5 & DEKFKKQYFYL & -7.1 \\
& Cyclotraxin B & CNPMGYTKEGC & -35.8 \\
\hline
\end{tabular}

PEP2 and Cyclotraxin B had 11, 17 and 14 hydrogen bonds with TrkB respectively. The proposed positioning of PEP1 and PEP2 on TrkB are shown in Fig. 2. Further details of these interactions obtained from LIGPLOT package can be found in the Supplementary Data. The result of molecular dynamics simulation indicated that both PEP1-TrkB and PEP2-TrkB complexes are stabilized after almost $4 \mathrm{~ns}$ simulations. The RMSD plot of PEP2-TrkB was even more acceptable than PEP1-TrkB and Cyclotraxin B-TrkB plots (Fig. 3). The two best peptides were purchased from TAG Copenhagen (Copenhagen, Denmark).

\section{Cytotoxic Effect of Designed Peptides on Ovarian Cancer Cell Lines}

For assessment of cytotoxic effect of selected peptides, two ovarian cancer cell lines including ov-car-3 and sk-ov-3 were treated with selected inhibitory peptides. The $50 \%$ inhibitory concentration (IC50) of PEP1 and PEP2 were calculated by performing MTT assay (Table 4). The IC50 of PEP2 was lower than Cyclotraxin B indicating higher inhibitory potential of PEP2 against TrkB receptor. 
Table 3 Residues of designed peptides interacting with TrkB as predicted by LIGPLOT analysis

\begin{tabular}{lll}
\hline $\begin{array}{l}\text { Receptor/ } \\
\text { inhibitor }\end{array}$ & Polar interaction forming AAs & Non-bonded contacts forming AAs \\
\hline TrkB & Lys333, Thr337, Lys312, Lys364, Leu324, Cys331, Lys328, & $\begin{array}{c}\text { His335, Thr332, Ile334, Pro313, Val307, Ala314, Leu315, } \\
\text { Gln316, Trp317, Asn325, Glu326, Gln347, Ile330 } \\
\text { Asp349, Tyr329 }\end{array}$ \\
PEP1 & Leu3, Asp4, Asp6, Asn11, Tyr9, Thr1 & Gly, Gln10, Gly7 \\
TrkB & Ser297, His299, Asn350, Asp349, Asp298, Gln347, Lys333, & Glu326, Ala314, Val307, Tyr342, Ile334, Cys331, Tyr329, \\
& Ser327, Thr332, Pro313, Lys312, Leu315 & Lys328, Ile330, His300 \\
PEP2 & Asn1, Thr2, Asp3, Leu5, Asn6, Asn8, Asn10, Gly11, Asp9 & Ser7, Leu4 \\
TrkB & His335, Lys333, Asp349, Lys328, Gln347, Cys331, Glu326, & His300, Thr337, Thr340, Tyr342, Val307, Pro313,Ala363, \\
& Leu315, Lys312, His339, Gly309 & Ala314, Ile334, Thr332, Ser327, Ile330, Tyr329 \\
PEP3 & Gln11, Asp10, Thr8, Gln6, Asn5, Gln3, Tyr2, Gln1 & Leu9, Asn4, Ala7 \\
TrkB & Ala314, Ile362, Asn325, Ser327, Ile330, Glu326, Lys328, & Pro313, Lys364, Phe305,Tyr34, Gly344, Ile334, Lys333, \\
& Leu315, Lys312 & Thr332, Cys331, Ile323, Gln316, Ala363 \\
PEP4 & Leu11, Gln7, Lys5, Asp1, Lys3 & Phe4, Glu2, Phe9, Tyr10 \\
TrkB & Asp349, Gln347, His300, His343, Ile334, Cys331, Lys333, & Lys364, Trp317, Asn325, Lys312, Lys328, Thr3, Ser327, \\
& Pro313, Glu326, Leu315, Leu324, Ala314, Gln316 & Gly344, Thr332, Phe305, Leu348 \\
PEP5 & Ser2, Ser1, Asp5, Gln4, Thr6, Gln8, Arg7, Ser11 & Asn9, Thr10, Thr3 \\
TrkB & Thr332, Ile334, Lys312, Gly344, Val307, Tyr342, Pro313, & Lys333, His343, Phe305, Thr306, Ala314, Gln316, Leu346, \\
& Leu315, Trp317, Leu324, Asn325, Glu326 & Ile323, Cys331 \\
Cyclotraxin B & Asn2, Cys1, Met4, Tyr6, Thr7, Cys11 & Glu9, Lys8, Pro3, Gly5, Gly10 \\
\hline
\end{tabular}

Fig. 2 Positioning of a PEP1, b PEP2 on the TrkB (Cyan) and hydrogen bonds of PEP1 (c) and PEP2 (d) with TrkB. The peptide inhibitors are shown in the red color. Schematic representation of hydrogen bonds between peptides and TrkB are shown in black dotted lines. For simplicity, any nonbonded contacts of TrkB with peptides have been omitted. TrkB residues involved in hydrogen bonding with peptides are indicated in green color (Color figure online)
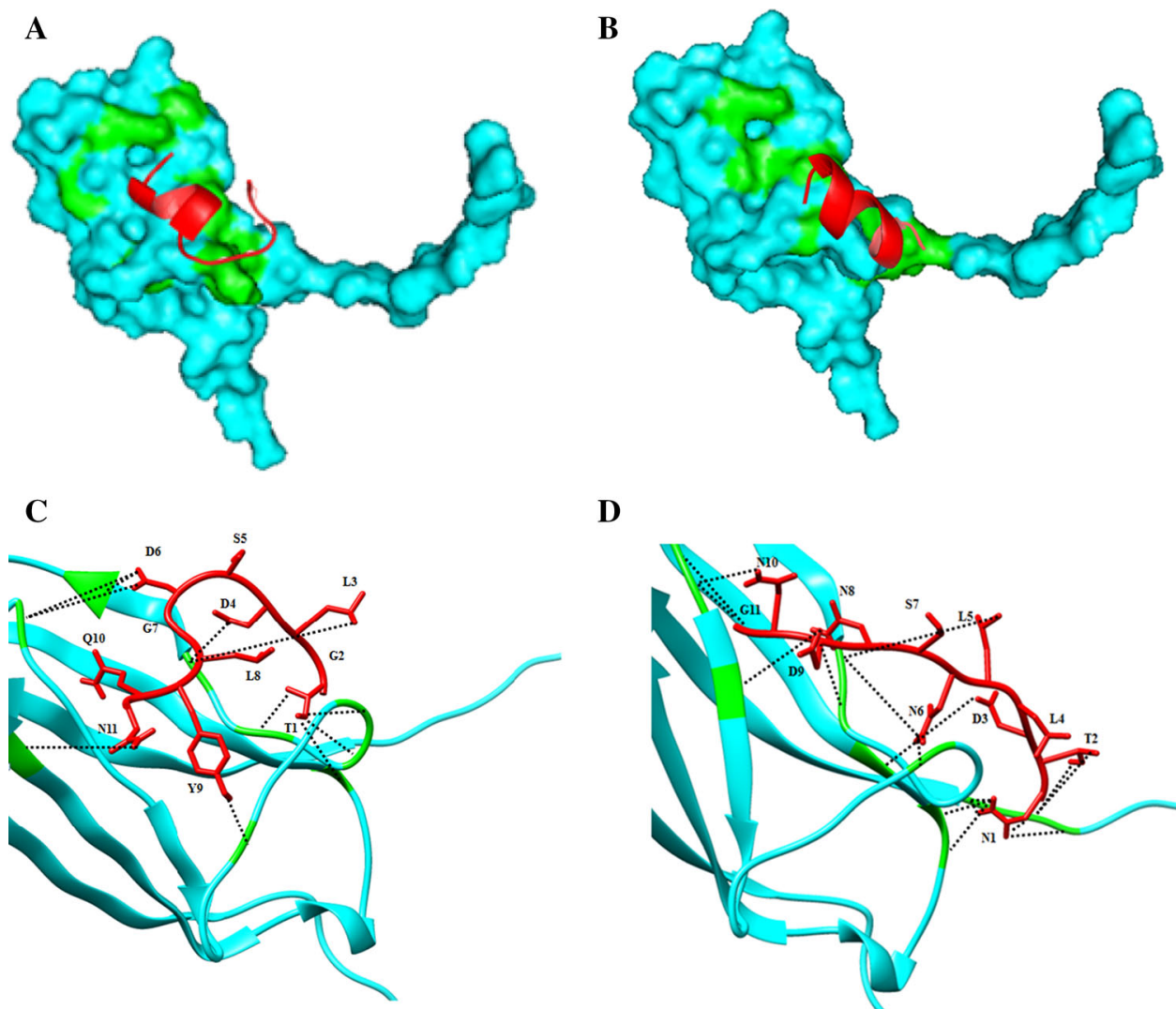

D

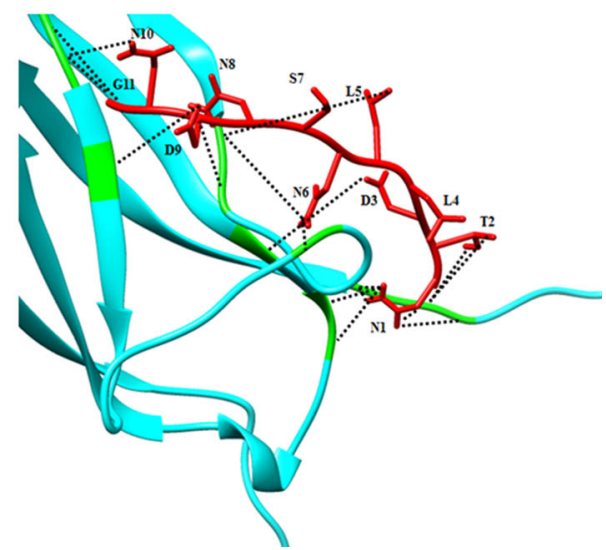



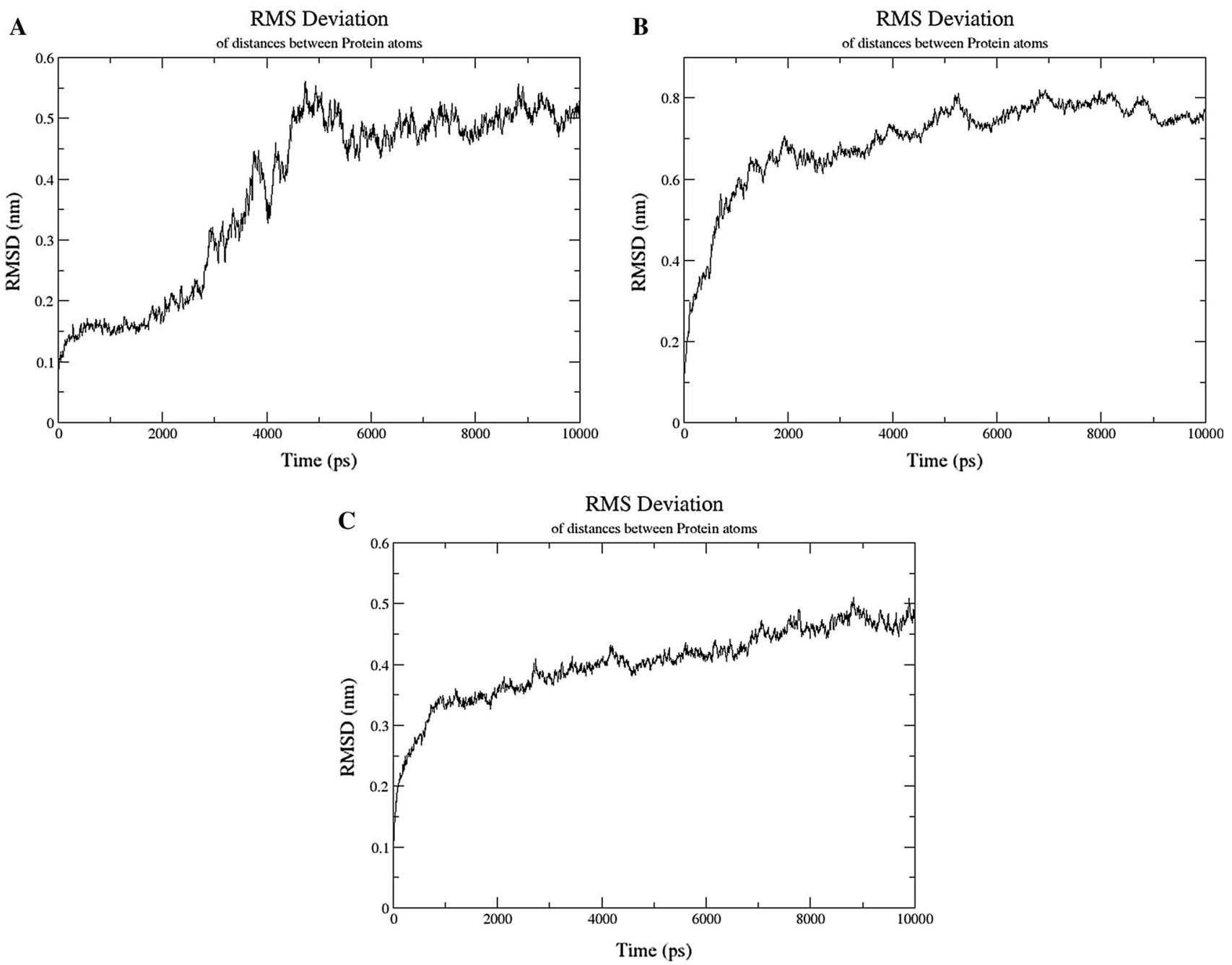

Fig. 3 The RMSD plot of a PEP1-TrkB, b PEP2-TrkB and c Cyclotraxin B-TrkB complexes simulation. The complexes were subjected to a $10 \mathrm{~ns}$ MD simulation with an integration time step of $0.002 \mathrm{ps}$

However, after $24 \mathrm{~h}$ treatment of the cells, the IC50 of PEP1 was clearly higher than Cyclotraxin B. Treatment of two ovarian cancer cell lines with inhibitory designed peptides reduced the cell viability significantly (A P value $<0.05$ was considered significant). Figure 4 summarizes the effect of PEP1 and PEP2 on ov-car-3 and sk-ov-3 cell

Table 4 The IC50 of tested inhibitory peptides

\begin{tabular}{lllll}
\hline Cell line & Peptide name & \multicolumn{3}{l}{ IC50 (nM) } \\
\cline { 3 - 5 } & & $24 \mathrm{~h}$ & $48 \mathrm{~h}$ & $72 \mathrm{~h}$ \\
\hline Ov-car-3 & PEP1 & 340.92 & 199.5 & 199.21 \\
& PEP2 & 199.52 & 198 & 197.3 \\
& Cyclotraxin B & 200 & 200 & 200 \\
Sk-ov-3 & PEP1 & 351.02 & 199.2 & 199.4 \\
& PEP2 & 199.45 & 197.2 & 198 \\
& Cyclotraxin B & 200 & 200 & 200 \\
\hline
\end{tabular}

viability with range $50-500 \mathrm{nM}$ of peptide concentrations after 24,48 and $72 \mathrm{~h}$ treatment.

\section{Apoptosis Evaluation in the Presence of Designed Inhibitory Peptides}

We evaluated the effect of PEP1 and PEP2 on the apoptosis of ov-car-3 and sk-ov-3 cell lines. After treatment of cells with concentration of 350 and $450 \mathrm{nM}$ designed peptides for 24 and $48 \mathrm{~h}$, we observed slight elevation in the amount of apoptotic cells. Approximately in both 24 and $48 \mathrm{~h}$ after the treatment with concentration of 350 and $450 \mathrm{nM}$ peptides, the apoptotic cells were obviously increased. Further details are shown in Table 5. The highest effect of peptides on the induction of apoptosis were observed in the ov-car-3 cell line compared with sk-ov-3 cell line, especially after $24 \mathrm{~h}$ treatment with PEP1 (31.59 and $32.21 \%$ apoptotic cells at concentration of 350 and $450 \mathrm{nM}$ respectively). These results confirm that the designed inhibitory PEP1 
Fig. 4 The effects of PEP1 and PEP2 on the ov-car-3 and sk-ov3 cell viability. The viability of two ovarian cancer cell lines reduced at different concentrations (50-500 nM) of designed peptides after 24,48 and $72 \mathrm{~h}$ treatment. $\mathbf{a}$ and $\mathbf{b}$ ovcar-3 cell line, $\mathbf{c}$ and $\mathbf{d}$ sk-ov-3 cell line. Control neg: untreated cells, Control pos: Cyclotraxin B-treated cells (Color figure online)
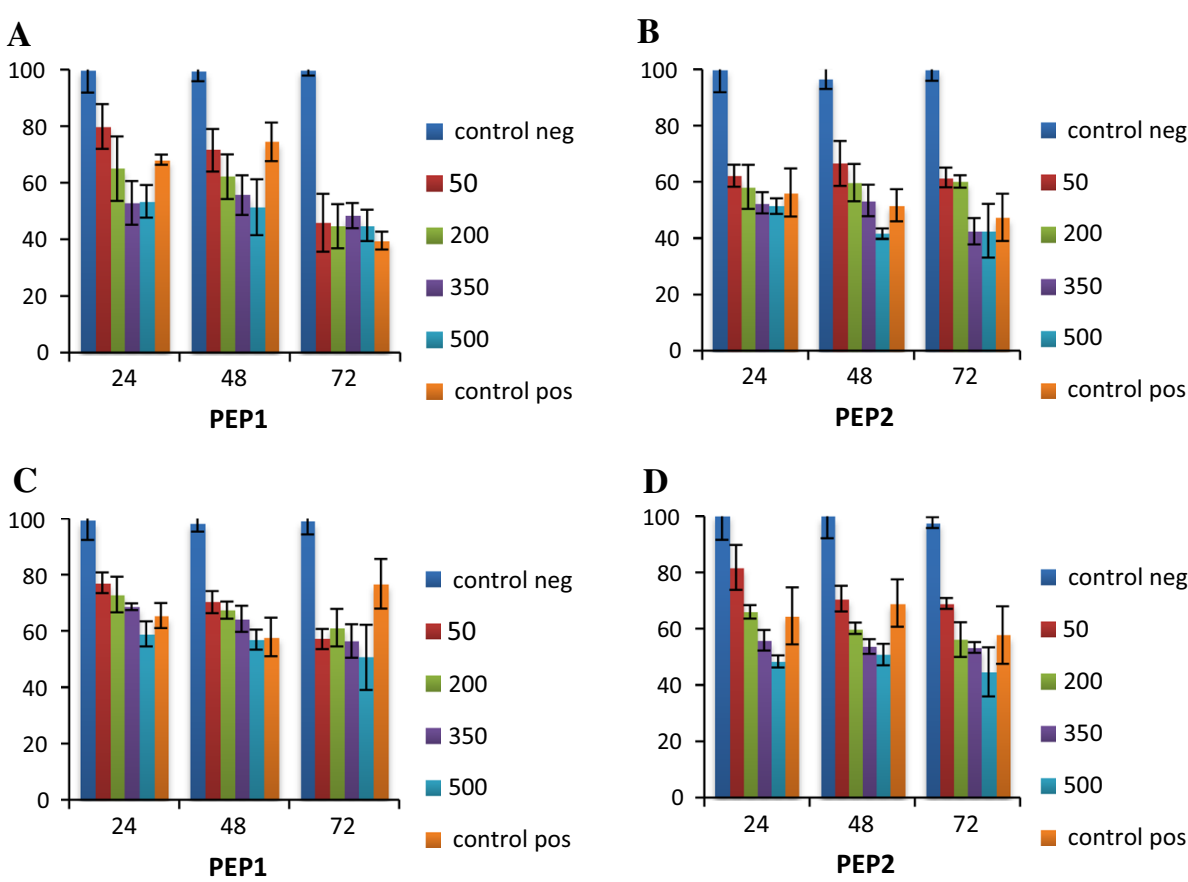

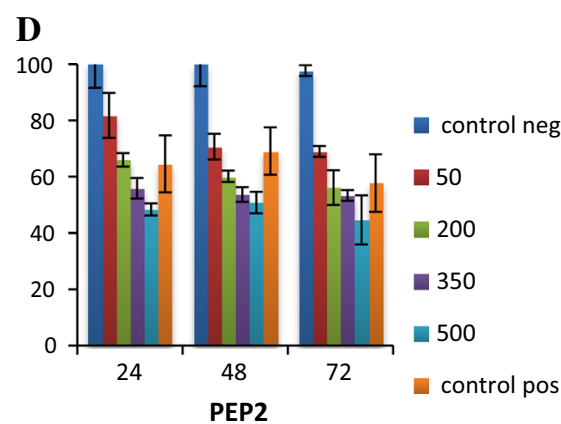

Table 5 Apoptosis percentage of treated cells with different concentrations of PEP1, PEP2 and Cyclotraxin B

\begin{tabular}{llllc}
\hline Cell line & Peptide name & Peptide concentration $(\mathrm{nM})$ & \multicolumn{2}{c}{ Apoptosis percentage $(\%)$} \\
\cline { 3 - 5 } & & $24 \mathrm{~h}$ & $48 \mathrm{~h}$ \\
\hline Ov-car-3 & PEP1 & 350 & 31.59 & 17.9 \\
& & 450 & 32.21 & 24.99 \\
& PEP2 & 350 & 23.18 & 20.79 \\
& & 450 & 25.33 & 28.39 \\
& Cyclotraxin B & 200 & 19.41 & 20.85 \\
& Negative control & & 2.82 & 2.82 \\
Sk-ov-3 & 350 & 18.07 & 20.11 \\
& PEP1 & 450 & 22.18 & 25.14 \\
& & 350 & 14.67 & 23.98 \\
& PEP2 & 450 & 24.22 & 23.53 \\
& & 200 & 20.56 & 16.47 \\
& Cyclotraxin B & & 2.32 & 2.32 \\
\hline
\end{tabular}

and PEP2 are capable of inducing apoptosis in the ovarian cancer cells.

\section{Analysis of TrkB Downstream Signaling Pathways}

The main importance of BDNF/TrkB signaling is that this signaling can regulate the function of some proliferationrelated proteins such as MAPKs and eIF4E through directly phosphorylation of these proteins. Western blotting result demonstrated that phosphorylation of TrkB, MAPK3 and eIF4E proteins declined in the treated cells when compared to control cells (Fig. 5). However, the total level of these proteins in the treated cells did not reduce significantly. The inhibition effect of PEP1 and PEP2 on the phosphorylation of aforementioned proteins, especially on the TrkB, were considerably higher than Cyclotraxin B. All three peptides (PEP1, PEP2 and Cyclotraxin B) reduced TrkB phosphorylation significantly. In addition, $\mathrm{PEP} 2$ also caused a significant reduction in eIF4E phosphorylation.

\section{Discussion}

In contrast to larger molecules, small peptide-based drugs would represent more appropriate treatment in cancer therapy because of their smaller size, excellent tissue 

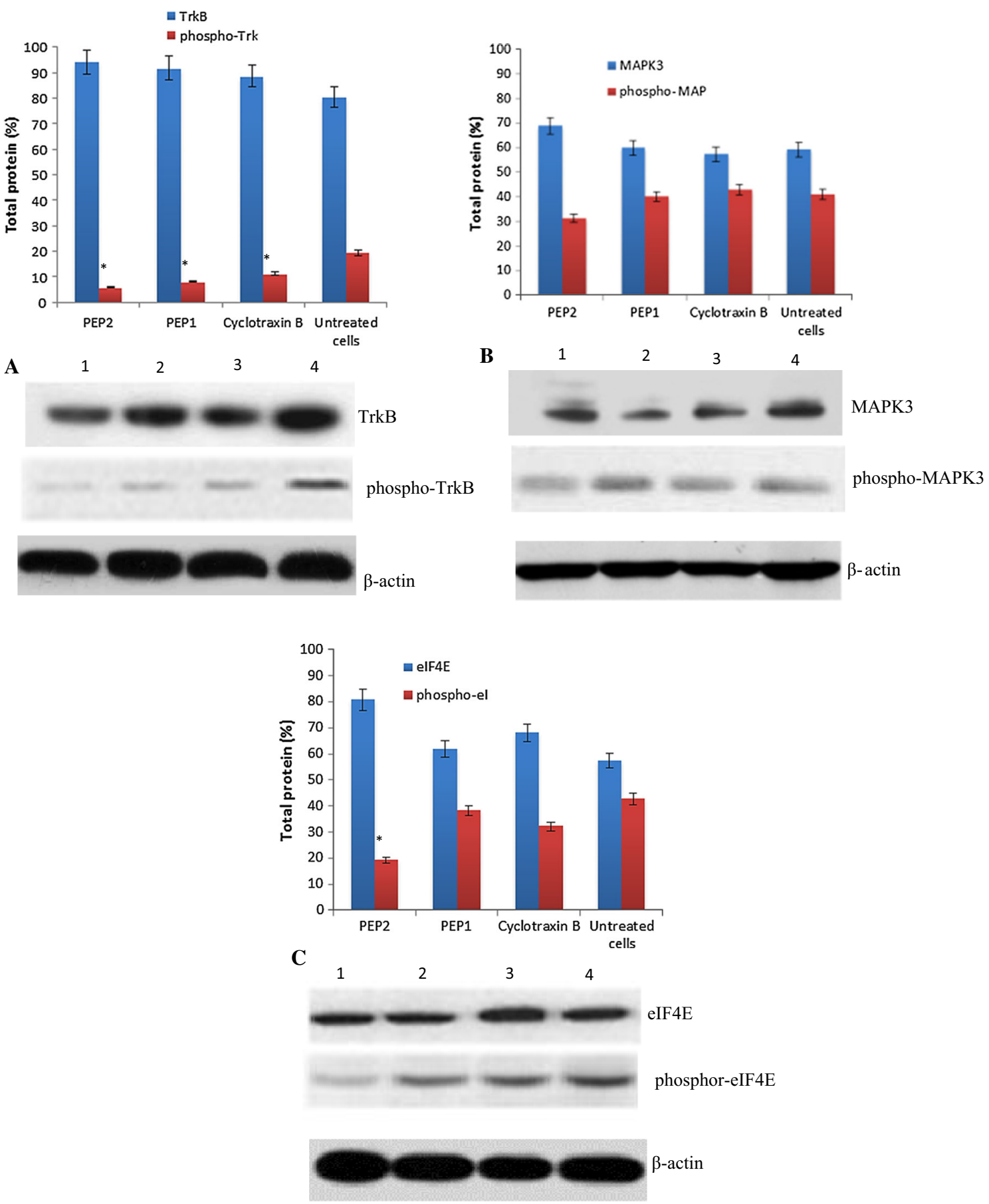

Fig. 5 Western blot analysis of total and phosphorylated level of TrkB and its downstream genes. a ( $U p$ anti-TrkB antibody, down antiphosphoTrkB antibody), b (up anti-MAPK3 antibody, down antiphosphoMAPK3 antibody), c (up anti-eIF4E antibody, down antiphosphoeIF4E antibody). Lane $1 \mathrm{PEP} 2$, lane $2 \mathrm{PEP} 1$, lane 3

penetration and easy synthesis process. There are several small peptides, also known as cell-penetrating peptides, that unlike other biotherapeutic agents can efficiently translocated through the plasma membrane. These peptides are widely considered as one of the most promising tools
Cyclotraxin B, lane 4 untreated cells. $\beta$-actin expression levels in the sample were used as the loading control. Blots were quantified and shown above each panel. PEP2 reduced phosphorylation of TrkB and eIF4E significantly $(P$ value $<0.05)$ (Color figure online)

for delivering numerous types of drugs (such as smallmolecule pharmaceuticals, therapeutic proteins, and antisense oligonucleotides) into target cells (Stewart et al. 2008). One of the most important challenges in peptidebased drug design is to find a promising therapeutic target 
associated with cancer progression. It has been shown that $\mathrm{BDNF} / \mathrm{TrkB}$ signaling is involved in the pathogenesis of different cancers mainly in ovarian cancer (Au et al. 2009) multiple myeloma (Hu et al. 2007) and colon cancer (Yu et al. 2010). Also, overexpression of TrkB has been reported in several human malignant tumors such as neuroblastoma which is likely to play an unfavorable role in neuroblastoma resistance to chemotherapy-induced apoptosis (Jaboin et al. 2002). There are some of the TrkB inhibitors which effectively inhibit BDNF/TrkB signaling in cancer cells and subsequently prevent cancer cell invasion (Desmet and Peeper 2006). AZ623, a TrkB inhibitor, in combination with another chemotherapy drug (topotecan) at concentration of 0.8 to $7 \mu \mathrm{M}$ could inhibit TrkBmediated signaling in neuroblastoma (Zage et al. 2011). Cazorla et al. indicated that the concentration of $200 \mathrm{nM}$ Cyclotraxin B efficiently inhibited TrkB receptor in vivo (Cazorla et al. 2010). In this study, we considered Cyclotraxin $\mathrm{B}$ as positive control. We introduced new potentially small peptide-based inhibitors of TrkB which could strongly modulate BDNF/TrkB signaling, even at lower concentrations compared to Cyclotraxin $\mathrm{B}$. The results of molecular docking studies revealed that PEP2 had a moderately strong Hydrogen-Bond pattern which results in a tight interaction with TrkB. The Asn1 residue of PEP2, by making a 6 hydrogen-bonds pattern with Ser297, Asp298, His299, Asp349 and Asn350 residues of TrkB, plays a very important role in the interaction of PEP2 with TrkB (Fig. 6). Generally, asparagine has potential hydrogen bonding atoms and plays a critical role in many enzymes functions and also helps protein refolding by interacting, directly or indirectly, with the hydrophilic groups (Choma et al. 2000). There are four asparagine residues in the sequence of PEP2 which may make a better binding affinity of this peptide to TrkB receptor.

The results of molecular docking revealed that the Lys333 residue of TrkB plays a key role in the binding of peptides to the TrkB. The Lys333 residue, in Cyclotraxin B-TrkB complex, makes a non-bonded contact with TrkB, while this residue makes two hydrogen bonds with both PEP1 (with Leu3 and Asp4 residues) and PEP2 (with Leu5 and Asn6 residues). It is now well established that a combination of Leu with either Asn or Asp residues is highly effective in inhibiting protein degradation (CARO et al. 1989). Combination of Leu with Asp and Asn in PEP1 and PEP2 respectively, may enhance the therapeutic effectiveness of these peptides by improving their stability.

Characterization of ligand-binding domain of the Trk family members have demonstrated that the hydrophilic residues in the Trk family members play a key role in the binding of ligands to Trk kinases (Ultsch et al. 1999). Similarly, the main candidate region of BDNF involved in the interaction with its receptor is surrounded by charged and

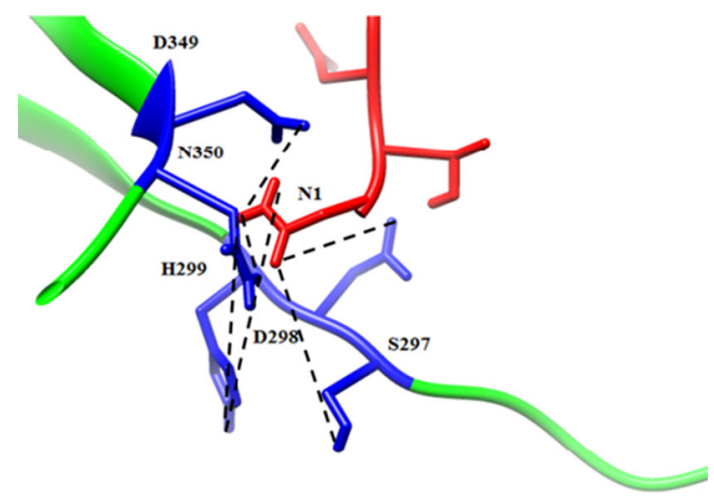

Fig. 6 The six hydrogen-bonds pattern of Asn1 residue of PEP2 (red color) interacted with TrkB. The residues of TrkB involved in the interaction with Asn1 residue of PEP2 are shown in blue color (Color figure online)

specificity-conferring residues (Robinson et al. 1999). These studies indicate that the binding of BDNF to TrkB is highly dependent on polar interactions between hydrophilic residues. The physicochemical properties of selected peptides and Cyclotraxin B calculated by Peptide property calculation tool of Innovagen (http://www.innovagen.se) showed a remarkable hydrophilicity scale of PEP2 which might explain better binding capacity of PEP2 than PEP1 and Cyclotraxin B (data not shown). Furthermore, the IC50 values of PEP2 were lower in 24, 48 and $72 \mathrm{~h}$ than Cyclotraxin B in the two treated ovarian cancer cell lines. The lower IC50 values indicate the greater potency of PEP2 in antagonizing the BDNF/TrkB signaling. Analysis by Western blotting indicated that PEP2 could significantly inhibit the phosphorylation of TrkB and its downstream genes.

TrkB triggers some downstream signaling pathways associated with cell growth and development. Activation of MAPKs cascade can be achieved by Trk receptor activation and the vast majority of evidences have demonstrated that MAPKs activation could increase cell migration in non-invasive cancer cells (Krueger et al. 2001). There are several new strategies for design of improved therapeutic approaches based on MAPKs function model in the cell (De Luca et al. 2012). Recent studies have reported that mTOR signaling pathway acts as a node of convergence down-stream of BDNF/TrkB (Slipczuk et al. 2009). BDNF/TrkB-induced increase of mTOR stimulates synthesis of proteins through regulation of p70S6K and 4E-BP1 (Park et al. 2014). Indeed, activated mTOR phosphorylates and inactivates $4 \mathrm{E}-\mathrm{BP} 1$ by repressing its binding to eIF4E (Troca-Marín et al. 2012). Then, free eIF4E could bind to the eIF4G and eIF4A and form eIF4F active complex facilitating translation initiation. In this study, we found that the phosphorylation of MAPK3 and eIF4E declined after treatment of the cells with TrkB designed inhibitors. Among three evaluated peptides, PEP2 could significantly inhibit the phosphorylation of eIF4E but there was no significant reduction in the phosphorylation of 
MAPK3 after treatment with inhibitory peptides. On the basis of recent studies, it is reasonable that the inhibition of TrkB receptor would not be expected to significantly modulate the function of MAPK signaling pathway. MAPKs are involved in many biological processes in a wide range of eukaryotic organisms. MAPK signaling pathway, which functions as one of the cell hub signaling pathways, is regulated by many other signaling pathways such as Tumor necrosis factor (TNF) (Chen et al. 2007), hypoxia (Risbud et al. 2005) and RAS/RAF (Hatzivassiliou et al. 2010). There is a large-scale study that introduces a list of 57 different genes activating MAPK signaling pathway (Matsuda et al. 2003). However, our results are clearly compatible with previous studies that have found the reduction in phosphorylation of TrkB accompanied by reduced downstream genes phosphorylation (Cazorla et al. 2010).

Up-regulation of TrkB can promote cell proliferation (Heo et al. 2013) and chemotherapy resistance (Lee et al. 2012) through inhibiting apoptosis. Expectedly, inhibition of TrkB by designed peptides slightly increased the amount of apoptosis in the set of cell lines studied here. Since inhibition of TrkB cannot completely block the anti-apoptotic function of BDNF (Takeda et al. 2013), we observed only a slight increase in the apoptotic cell population.

In conclusion, the aim of present study was to demonstrate that inhibition of TrkB by peptide-based inhibitors can considerably down-regulate its downstream target genes including MAPK3 and eIF4E. In fact, the modulating of these genes may enhance the chemotherapy responses in cancer cells through regulation of cell death. Therefore, the TrKB inhibition by means of rational designed peptides would be useful in control of that pathway.

Acknowledgments This work was financially supported by Qazvin university of Iran; we also appreciate. Ali Ghafari for linux command setting.

Funding This study was funded by Qazvin University of Medical Sciences.

\section{Compliance with Ethical Standards}

Conflict of Interest The authors declare that they have no conflict of interest.

Ethical approval This article does not contain any studies with human participants or animals performed by any of the authors.

\section{References}

$\mathrm{Au}$ CW et al (2009) Tyrosine kinase B receptor and BDNF expression in ovarian cancers-Effect on cell migration, angiogenesis and clinical outcome. Cancer Lett 281(2):151-161

Caro LHP et al (1989) A combination of intracellular leucine with either glutamate or aspartate inhibits autophagic proteolysis in isolated rat hepatocytes. Eur J Biochem 181(3):717-720
Cazorla M et al (2010) Cyclotraxin-B, the first highly potent and selective TrkB inhibitor, has anxiolytic properties in mice. PLoS One 5(3):e9777

Chen S-E et al (2007) TNF- $\alpha$ regulates myogenesis and muscle regeneration by activating p38 MAPK. Am J Phys Cell Physiol 292(5):C1660-C1671

Choma C et al (2000) Asparagine-mediated self-association of a model transmembrane helix. Nat Struct Mol Biol 7(2):161-166

Dawson $\mathrm{H}$ et al (2014) Tyrosine kinase receptor B (TrkB) expression in colorectal cancers highlights anoikis resistance as a survival mechanism of tumour budding cells. Histopathology 66:715-725

De Luca A et al (2012) The RAS/RAF/MEK/ERK and the PI3 K/ AKT signalling pathways: role in cancer pathogenesis and implications for therapeutic approaches. Expert Opin Ther Targets 16(S2):S17-S27

De Vries SJ et al (2007) HADDOCK versus HADDOCK: new features and performance of HADDOCK2. 0 on the CAPRI targets. Proteins 69(4):726-733

Desmet C, Peeper DS (2006) The neurotrophic receptor TrkB: a drug target in anti-cancer therapy? Cell Mol Life Sci CMLS 63(7-8):755-759

Fujikawa $\mathrm{H}$ et al (2012) High TrkB expression levels are associated with poor prognosis and EMT induction in colorectal cancer cells. J Gastroenterol 47(7):775-784

Götz R, Sendtner M (2014) Cooperation of tyrosine kinase receptor TrkB and epidermal growth factor receptor signaling enhances migration and dispersal of lung tumor cells. PLoS One 9(6):e100944

Hatzivassiliou G et al (2010) RAF inhibitors prime wild-type RAF to activate the MAPK pathway and enhance growth. Nature 464(7287):431-435

Heo $\mathrm{H}$ et al (2013) Upregulation of TrkB by forskolin facilitated survival of MSC and functional recovery of memory deficient model rats. Biochem Biophys Res Commun 431(4):796-801

$\mathrm{Hu} \mathrm{Y}$ et al (2007) Identification of brain-derived neurotrophic factor as a novel angiogenic protein in multiple myeloma. Cancer Genet Cytogenet 178(1):1-10

Huang EJ, Reichardt LF (2003) Trk receptors: roles in neuronal signal transduction*. Annu Rev Biochem 72(1):609-642

Jaboin $\mathbf{J}$ et al (2002) Brain-derived neurotrophic factor activation of TrkB protects neuroblastoma cells from chemotherapy-induced apoptosis via phosphatidylinositol $3^{\prime}$-kinase pathway. Cancer Res 62(22):6756-6763

Jang S-W et al (2007) Gambogic amide, a selective agonist for TrkA receptor that possesses robust neurotrophic activity, prevents neuronal cell death. Proc Natl Acad Sci 104(41):16329-16334

Jiang M et al (2013) Small-molecule TrkB receptor agonists improve motor function and extend survival in a mouse model of Huntington's disease. Hum Mol Genet. doi:10.1093/hmg/ddt098

Krueger JS et al (2001) Temporal and quantitative regulation of mitogen-activated protein kinase (MAPK) modulates cell motility and invasion. Oncogene 20(31):4209-4218

Lee $J$ et al (2012) A novel role for BDNF-TrkB in the regulation of chemotherapy resistance in head and neck squamous cell. PLoS One 7(1):e30246

Li Y et al (2012) Conditional ablation of brain-derived neurotrophic factor-TrkB signaling impairs striatal neuron development. Proc Natl Acad Sci 109(38):15491-15496

Maina F (2014) Strategies to overcome drug resistance of receptor tyrosine kinaseaddicted cancer cells. Curr Med Chem 21(14):1607-1617

Matsuda A et al (2003) Large-scale identification and characterization of human genes that activate NF- $\mathrm{KB}$ and MAPK signaling pathways. Oncogene 22(21):3307-3318

Maupetit J et al (2009) PEP-FOLD: an online resource for de novo peptide structure prediction. Nucleic Acids Res. doi:10.1093/nar/ gkp323 
Nakagawara A (2001) Trk receptor tyrosine kinases: a bridge between cancer and neural development. Cancer Lett 169(2):107-114

Okamura K et al (2012) Expression of TrkB and BDNF is associated with poor prognosis in non-small cell lung cancer. Lung Cancer 78(1):100-106

Park SW et al (2014) Differential effects of antidepressant drugs on mTOR signalling in rat hippocampal neurons. Int J Neuropsychopharmacol 17(11):1831-1846

Pronk S et al (2013) GROMACS 4.5: a high-throughput and highly parallel open source molecular simulation toolkit. Bioinformatics. doi:10.1093/bioinformatics/btt055

R Development C (2012) TEAM 2009: R: a language and environment for statistical computing. Vienna. http://www.R-project.org

Rasband W (2012) ImageJ: image processing and analysis in Java. Astrophys Source Code Libr 1:06013

Risbud MV et al (2005) Hypoxia activates MAPK activity in rat nucleus pulposus cells: regulation of integrin expression and cell survival. Spine 30(22):2503-2509

Robinson RC et al (1999) The structures of the neurotrophin 4 homodimer and the brain-derived neurotrophic factor/neurotrophin 4 heterodimer reveal a common Trk-binding site. Protein Sci 8(12):2589-2597

Rohl CA et al (2004) Protein structure prediction using Rosetta. Methods Enzymol 383:66-93

Slipczuk L et al (2009) BDNF activates mTOR to regulate GluR1 expression required for memory formation. PLoS One 4(6):0006007

Stewart KM et al (2008) Cell-penetrating peptides as delivery vehicles for biology and medicine. Org Biomol Chem 6(13):2242-2255
Takeda K et al (2013) BDNF protects human vascular endothelial cells from $\mathrm{TNF} \alpha$-induced apoptosis. Biochem Cell Biol 91(5):341-349

Thiele CJ et al (2009) On Trk-the TrkB signal transduction pathway is an increasingly important target in cancer biology. Clin Cancer Res 15(19):5962-5967

Troca-Marín JA et al (2012) Deregulated mTOR-mediated translation in intellectual disability. Prog Neurobiol 96(2):268-282

Ultsch MH et al (1999) Crystal structures of the neurotrophin-binding domain of TrkA, TrkB and TrkC. J Mol Biol 290(1):149-159

Wallace AC et al (1995) LIGPLOT: a program to generate schematic diagrams of protein-ligand interactions. Protein Eng $8(2): 127-134$

Wu D et al (2014) Peptide-based cancer therapy: opportunity and challenge. Cancer Lett 351(1):13-22

$\mathrm{Yu} \mathrm{X}$ et al (2008) Suppression of anoikis by the neurotrophic receptor TrkB in human ovarian cancer. Cancer Sci 99(3):543-552

$\mathrm{Yu}$ Y et al (2010) Overexpression of TrkB promotes the progression of colon cancer. Apmis 118(3):188-195

Zage PE et al (2011) The selective Trk inhibitor AZ623 inhibits brainderived neurotrophic factor-mediated neuroblastoma cell proliferation and signaling and is synergistic with topotecan. Cancer 117(6):1321-1391

Zeng Y et al (2013) Small molecules activating TrkB receptor for treating a variety of CNS disorders. CNS Neurol Disord Drug Targets (Former Curr Drug Targets CNS Neurol Disord) 12(7):1066-1077 